\title{
Fabrication of Non-Implant 3D Printed Skin
}

\author{
Yong Leng Chuan*, and Shivani Amish Kumar Pandya \\ Faculty of Built Environment, Engineering, Technology \& Design, Taylor's University Lakeside \\ Campus, Malaysia.
}

\begin{abstract}
Many bandages tend to be harmful when being removed from the human skin. This is a crucial issue, especially faced by burn victims. When bandages are removed from the burn wound, they tend to be harmful by peeling off the newly formed layer of skin over the burn wound. Such nature causes the patient to endure a longer recovery time with additional pain. The objective of this project is to $3 \mathrm{D}$ print artificial skin for the victims of burn wounds by using natural gelation. The main aim for creating the artificial skin will be used in place of the current burn wound treatment techniques of dressing the wounds in bandages. The inner layer of this skin was lined with a natural adhesive, a thin layer of agar-agar, which has been reinforced with crushed eggshells to increase its adhesive strength and durability. The synthesized gel contained non adhesive behavior, yet aids in wound healing abilities. Applying hydrocolloids ensures that the wound is kept cool and the gel also ensures efficient heat transfer. This was done so that less sweating occurs on the patient. Based on the experiments that were conducted, the results conclude that the best ratio of artificial skin layer would be 2:1 of agar gel: crushed eggshells. This golden ratio of gel: crushed eggshells for the longest period of time for attachment on the skin without sweating, is achieved. The skin will be printed using Acrylonitrile-Butadiene-Styrene (ABS). The colour of the skin and the shape of the skin was individually designed for each specific patient. The inner gel has the capabilities of reducing the rehabilitation time, without compromising the comfort of the patient. This approach has the potential to be used as a new method to treat burn wounds..
\end{abstract}

\section{Introduction}

The main purpose of creating the $3 \mathrm{D}$ printed skin is to aid burn victims. According to the World Health Organization (WHO), there are an estimated of 265,000 fatalities every year caused by burns alone [1]. There are countless more people with burn injuries that are admitted into hospitals every year. Treatment for burn patients depends on the severity of the burn and how many layers of skin have been damaged from the burn. That will also determine the number of days the treatment will take and how many days the patient needs to be hospitalized.

\footnotetext{
Corresponding author: LengChuan.Yong@taylors.edu.my
} 
When victims have severe burn wounds, it will be a long and painful process to treat them all properly. Not all the skin layers can be treated at once; hence multiple surgeries have to be performed. The human skin has to be given some time to heal on its own before surgery can be performed again [2]. Whilst waiting for the patient to heal, the wound cannot be exposed to air and must be wrapped with gauze and bandages to prevent bacterial infections [3]. Despite this being a commonly practiced method that is used by hospitals worldwide, there are many accidents that are caused by the bandages themselves. When the bandages are removed from the area of the burn, it may cause tearing and stripping of the skin, which causes the burned skin to be further irritated, hence prolonging the recovery time. Based on a study of 155 residents in long-term care facilities who were at least 65 years of age, the incidence of new tape-related skin injuries was $15.5 \%$ over an 8 -week period with skin injuries developing at 34 sites in 24 patients [4].

For burn victims, plastic surgery is a luxury and is not affordable for everyone. It is an expensive procedure that may contain plenty of risks. Especially, in the case of a deep skin injury, multiple procedures are required to fix the wound and get the skin surface looking normal again by placing multiple layers of skin grafts. Non-implant skin can be an alternative to solve this issue. This can be used by people of all financial means and has significantly less risks compared to undergoing surgery. Non implant skin means that there is no surgery required as the skin will not be embedded into your body. This is a cheaper alternative and relatively safer than surgery. The non-implant skin can be reused and the gel created with natural substances is compatible with the human skin, which will also promote recovery [5]. Fortunately, medical technology constantly advances, creating better healthcare and opportunities for patients. New discoveries have been made in the field of $3 \mathrm{D}$ printing, where a $3 \mathrm{D}$ printer is used to print tissues samples and skin layers that can be implanted into the human body using stem cells. However, this too is still an experimental procedure and costs a lot of money [6]. There are two main reasons for creating 3D printed skin for burn victims. Firstly, there is for the purpose of covering up their scars after their burn wounds have been treated. Secondly, it can be used for the healing process of the burn victims. The second reason is what will be discussed in this report based on the healing of the wounds of the burn victims. This is because the gel layer synthesized has natural organic substances, all of which promote skin growth, which aids in the recovery time of the burn victims.

A 3D printer is used so that the skin can be easily customized to the different types of structures that each person has. 3D printing is an efficient option as it can cater to each patient's specific applications. It is also a fast prototyping method with high accuracy and low fabrication cost. The filament to be used for the $3 \mathrm{D}$ printer is the acrylonitrile butadiene styrene (ABS). ABS is used as the filament because it is a material that can be easily sterilized and that is easy to mould. With medical applications, sterilization is a very important factor [7]. Another crucial element for the non-implant 3D skin will be the gelatinous substance that will be used to attach the 3D printed skin to the patient's burnt skin. The gel will be synthesized using hydrocolloids which have excellent healing properties for the skin and have adhesive strength that will be able to replace the current adhesives in bandages.

This research is done to have an alternative method to apply to the current care of burn victims. Instead of using the bandages and any medical adhesives, a 3D printed skin mask is to be used to promote healing. Furthermore, the gel produced will be nontoxic and hence will not be an irritant to the skin. 


\subsection{Research Methodology}

\subsection{Raw materials for gel formulation}

The raw material that has been used to synthesize the inner layer of the artificial skin is agar, crushed eggshells and glycerol. The eggshells were roughly crushed using a pestle and mortar and were mixed with the agar. The reason for using roughly crushed eggshells compared to using eggshell powder is that the hand crushed eggshells contain the permeable membranes of an eggshell. The permeability of these membranes ensure that the entire solution remains "breathable". Agar has excellent rheological properties and hence have many applications in the biomedical industry. Agar is from a plant origin, along the walls of certain types of algae. The agar in the seaweed provides the flexible motion for the seaweed to be able to follow the current of the ocean [8]. The flexibility of agar can be useful in the project as the contours of the human body are not easy to adapt to and hence the flexibility will ensure the easy adhesion of the gel to the skin. The agar was then reinforced with crushed eggshells and glycerol.

The eggshell of a chicken egg contains $95 \%$ of calcium carbonate and $5 \%$ of organic materials such as collagen. Collagen is the main component in the extracellular matrix of all tissue types. It is biocompatible and is non-toxic. This collagen will be helpful in tissue regeneration [9]. It is useful for burn victims as it also would help speed up the recovery of the patient.

Another substance that was tested with was glycerol. A preliminary experiment conducted with plain agar and glycerol was also not particularly effective in showing good spreading.

During the formulation, the crushed eggshell powder was added to the boiled agar solution and blended homogeneously. This was tested in different concentrations to see which one was the optimum solution. Secondly, the eggshell powder was added to the solution along with glycerol. This was then compared with the solution of agar and eggshells. The best synthesized gel was chosen from these experiments.

\subsection{Breathability}

Breathability is one of the crucial factors of this project. The gel that is created should be "breathable". This is to ensure that no sweating occurs between the gel layer and the human skin. If there is sweat that pools up between the two layers, it will cause slippage of the artificial skin and hence would reduce the duration that the skin can remain attached to the human skin. Agar is a permeable substance that allows diffusion to occur. This will ensure that the sweating of the skin will not pool. Furthermore, all the additives that are tested with to create the gel, are permeable. The eggshells are used in crushed form instead of powder form because when the eggshells are crushed, the inner and the outer membrane is also included in the mixture. Both the inner and out membrane are permeable. This is added to the mixture when creating the gel. Furthermore, glycerol is also a substance that permeable. Hence, when the gel mixture is created, it is ensured that there is low risk of slippage of the artificial skin. Moreover, there ABS that is used to print the artificial skin also has the properties of being water permeable. This means that the sweat that would occur would have room to escape and would not affect the gel layer. 


\subsection{Determination of gel : crushed eggshells ratio}

The aim of this step is to determine the best spreading and adhesion by adding crushed eggshell powder and glycerol to the agar gel. The factors that will be considered in the synthesizing of the gel is the concentration of the crushed eggshell powder that is put in the agar gel. The experiment was conducted by testing the gel and 3D printed skin on a test subject. The time taken for the gel to dry and the time that the printed skin remained stuck to the human skin were both measured.

The aim of this experiment is eventually to determine the best combination of the three raw materials - agar, crushed eggshells and glycerol, to be able to produce a gel that is quick to dry on the skin and has the ability to stick to the human skin for longer periods of time. Figure 1 below shows the raw materials used while Figure 2 shows the mixture of the substances.

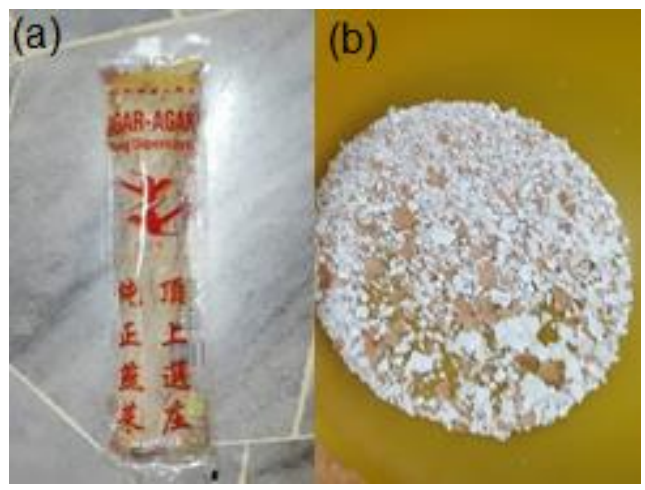

Fig. 1. (a) Dry Agar. (b) Crushed Eggshells

(a)

(b)

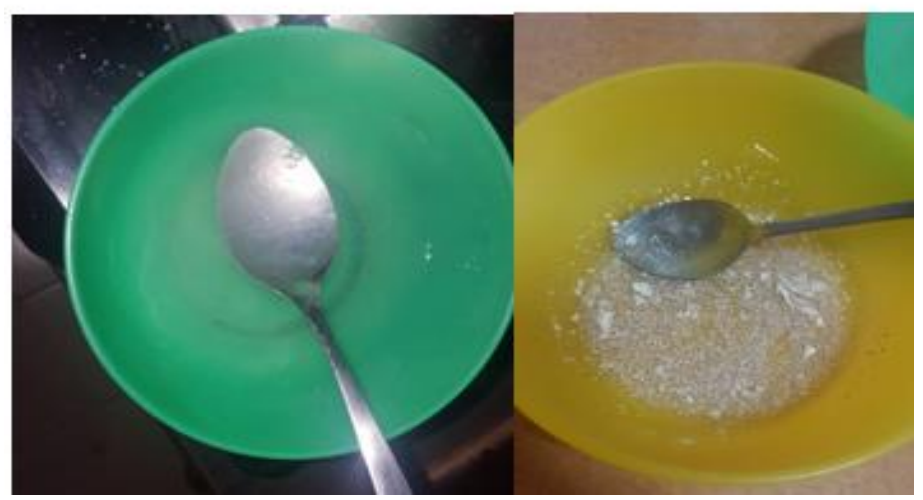

Fig. 2. (a) Combination of agar gel and glycerol. (b) Combination of agar gel, glycerol and eggshells.

The results for this experiment were found by applying a layer of the gel synthesized on the $3 \mathrm{D}$ printed skin. This $3 \mathrm{D}$ printed skin is held in place until the solution is dry on the human skin. The time taken for the solution to dry on the human skin is measured and then the time the skin remains on the human skin is also measured and recorded. The thin layer of the gel spread on the 3D printed skin and how the 3D printed skin was tested on the human skin is shown in the Figure 3 below. For this research, there was only a single test 
subject as the concept was trying to be proven where this new research could be a viable alternative in place of bandages. To be able to incorporate this into the medical field, further testing is required on more skin types.

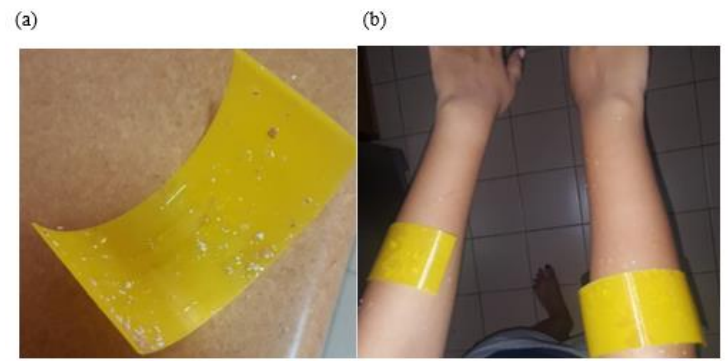

Fig. 3. (a) A layer of synthesized gel (agar, crushed eggshells and glycerol) applied on the 3D printed skin. (b) The adhesion testing of the 3D printed skin on the human skin

\subsection{D Printed Artificial Skin Outer Layer}

To design the 3D printed skin, measurements were taken of an actual test subject and the skin was printed to fit the user's arm perfectly. Once the measurements of the subject's arm were taken, design was modelled using Solidworks. The material selected to print the skin was ABS. Figure 4 below shows the customized arm design of the printed skin in Solidworks.

(a)

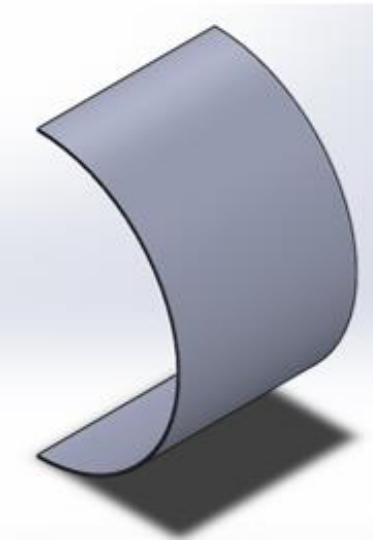

(b)

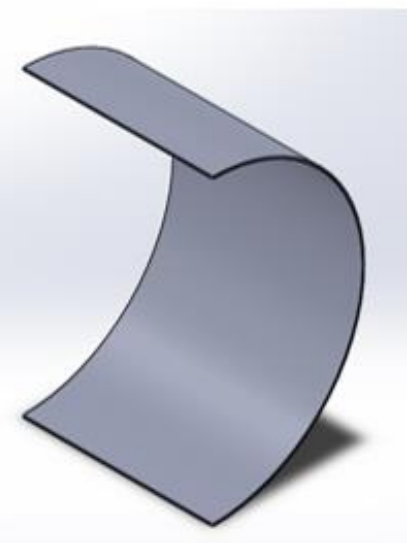

Fig. 4. The arm design of the $3 \mathrm{D}$ printed skin

The measurements of the artificial skin had the height of $8 \mathrm{~cm}$ and the width of $5 \mathrm{~cm}$, with the radius of curvature of $4 \mathrm{~cm}$. The thickness selected for the skin was $0.5 \mathrm{~mm}$. This is selected as the thickness of the skin as the actual thickness of human skin is $3 \mathrm{~mm}$ and the aim was to ensure the printed skin was light enough to not be able to be felt by the user and flexible enough to fit the contours of the human body, without being too fragile to break. Figure 5 below shows the actual printed skin. 
(a)

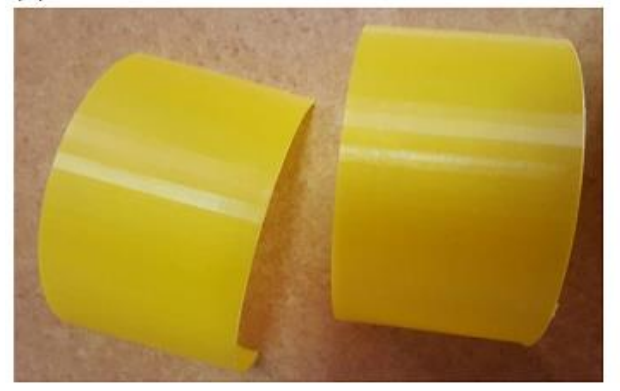

(b)

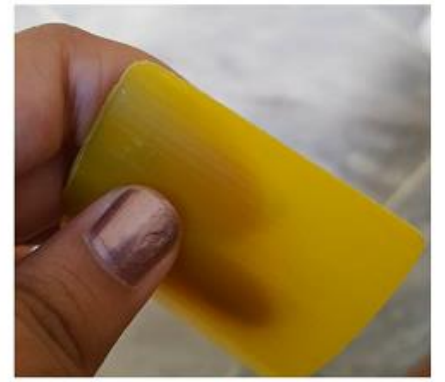

Fig. 5. (a) The 3D printed skin for the arm, (b) The 3D printed skin for the arm with rounded edges

The issue of safety always arises when creating something for human usage. This is why, special consideration was made when creating the skin. It was initially printed as a rectangular piece with sharp edges to ensure the dimensions taken from the test subject were correct. Once the printed piece was fitted to check if the skin fits the wound, the edges of the skin were filed to ensure that the sharp edges do not do further damage to the skin.

\subsection{Results and Discussion}

There are two experiments that were conducted. The first experiment tests which mixture is optimum for the gel synthesis. The second experiment tests the different concentrations of eggshells to see how the varying concentrations of eggshells affect the adhesive strength of the gel. A preliminary experiment was conducted to minimize the mixtures to be tested. The initial experiment tested the time taken for the mixture to dry on the human skin along with the duration it adhered to the human skin. The agar gel with the glycerol takes the shortest time on average to completely stick the paper on the human skin. Whilst plain agar had a lower time to dry completely, the adhesiveness of agar was weaker than that of the rest of the mixtures. This means that while doing routine work with the arm, the paper detached itself from the human skin. This means that the adhesive strength of plain agar is too weak to be able to allow the artificial skin onto the human skin if it cannot support a piece of paper to stay stuck to the human skin. Similarly, for the glycerol and the agar mixture, the mixture has very low adhesiveness. Since both the substances are water based, it has low stickiness and the paper slips very easily when some force is applied on it. Hence, for further experiments, the plain agar and the agar with glycerol mixture were not considered and only the other two mixtures were tested.

The reason for the agar and the glycerol mixture to dry faster compared to the neat agar is because both glycerol and agar are water based solutions, which has lower viscosity compared to the neat agar. The addition of glycerol to the neat agar makes it a more watery solution and hence has less adhesiveness and the ability to dry quicker.

\subsection{Gel formation theory}

The first experiment conducted was to compare the two different mixtures that are tested to synthesize the inner gel. The first experiment measures the time taken for the mixture to dry on the human skin and the duration for which it remains attached on the skin. The results showed when the two different mixtures are applied to the 3D printed surface and given 
time to allow them to continue adhering to the human skin. Figure 7 below represents the duration for which the printed skin remained adhered to the human skin.

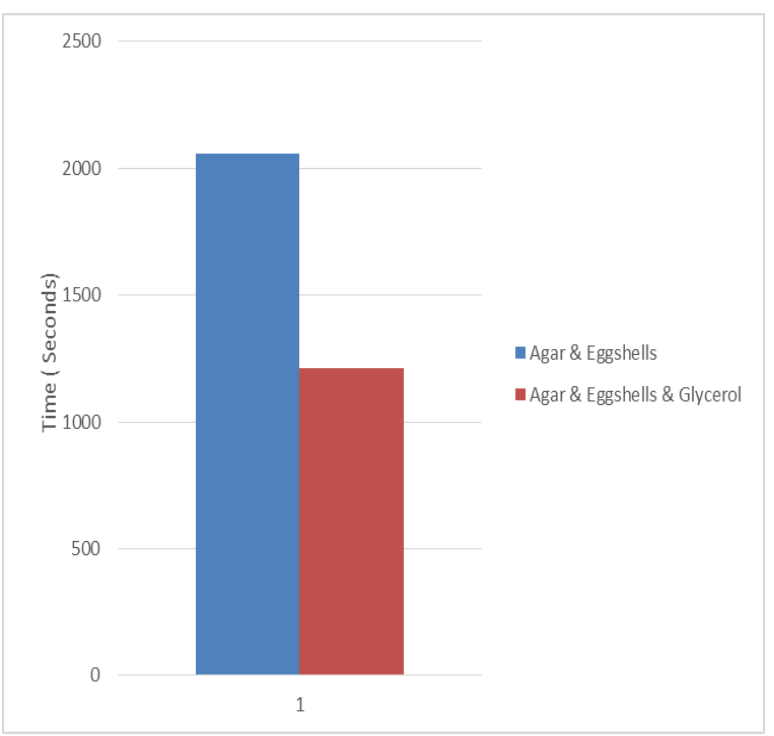

Fig. 7. Graph representing Experiment 1

From the results obtained in the first experiment that the more effective solution to be used as the gel is the agar and the eggshells when compared to the agar, eggshells and the glycerol. The agar and eggshells solution takes a lesser time to dry and has the capabilities of remaining on the human skin for a significantly longer period of time. This results were obtained after conducting the experiment twice and taking the average reading for more accurate results. The experiment is also conducted at the same time period and in the same area so there are no environmental factors that affect the results of the experiment.

\subsection{Gel Adhesion Testing based on different crushed eggshells composition}

Another factor that can affect the duration for which the synthesized gel can remain on the human skin is the percentage of eggshells that are added into each mixture. Hence, the gels are tested again with different concentrations of eggshells to be able to see which concentration is optimum to be used for the gel synthesis. The results below shows that the experiment conducted when different concentrations of eggshells were used for both the separate mixtures. Figure 8 shows the graphical representation of the results obtained. 


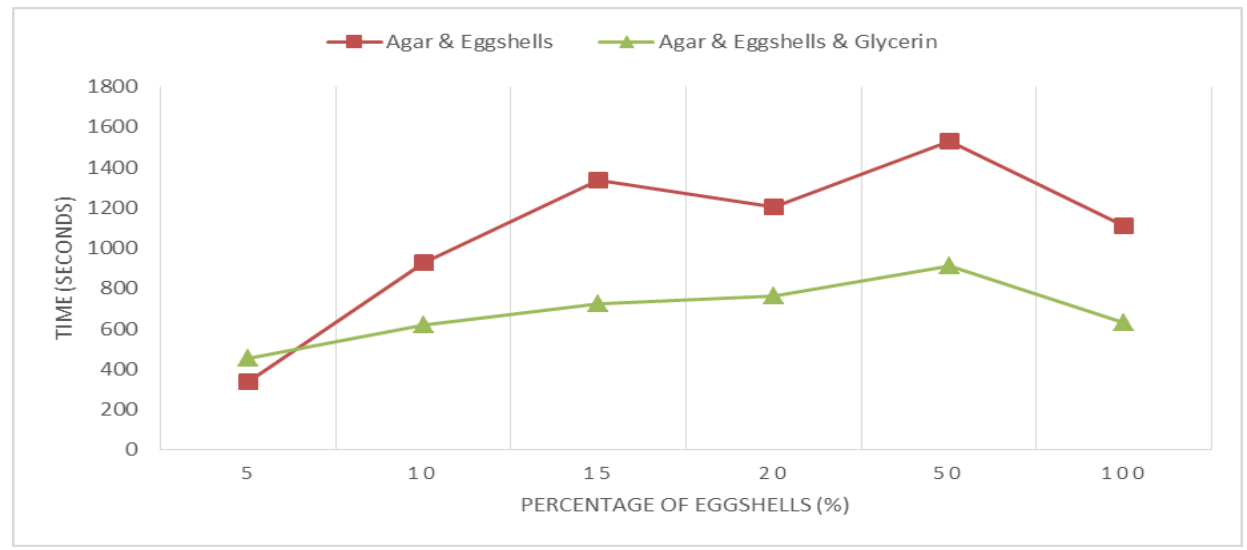

Fig. 8. Graph representing Experiment 2

It can be concluded from the results obtained in the second experiment that the optimum concentration of eggshells that should be used for the gel is $50 \%$. The agar with the $50 \%$ concentration of eggshells has the longest duration of time that the printed skin was stuck to the human skin. When compared between the two mixtures, the longest duration that the mixture with glycerol is 15 minutes, whereas the longest duration that the mixture without glycerol is about 1.6 times more than the mixture with glycerol at 25 minutes. Hence, it can be said that the mixture that should be used to synthesize the gel should be agar gel with $50 \%$ eggshells.

It can be observed that at $20 \%$ of eggshells concentration, there is an anomaly in the results. It can be seen that at the $20 \%$ concentration of crushed eggshells, the duration of it sticking to the human skin decreases. This is due to some inconsistencies in the experimental results. This is why it does not follow the trend of the overall graph.

At $100 \%$ of eggshells, the boiled agar solution is already saturated with the crushed eggshells and hence does not produce more efficient results in terms of the duration of time it is allowed to stick on the human skin. The trend of the graph is observed closely, hence it can be deduced that the optimal percentage of eggshells to be added to the boiled agar solution is $50 \%$ of eggshells, without the additional glycerol.

The results show that the better solution to use as the gel is the agar and crushed eggshell solution without the usage of glycerol. This is because the usage of glycerol causes slippage of the artificial skin. The addition of the glycerol makes the solution reduce in adhesive strength. The adhesive strength is measured on the basis of the duration the artificial skin can adhere to the human skin without any external applied force. For the purposes of this experiment, the adhesiveness is not tested based on the amount of force each mixture can handle. This is why, the selected solution for spreading on the artificial skin is agar and $50 \%$ of crushed eggshells. 


\subsection{Conclusion}

The optimum ratio of gel: crushed eggshells is $2: 1$. This ratio showed that the ability of the $3 \mathrm{D}$ printed outer layer to adhere to the human skin has been achieved. Using the optimum ratio, there was a short drying time along with it being able to adhere to the human skin for a long duration of time. Furthermore, the artificial skin showed no adhesive stains or marks after the skin was peeled off the human skin. This is beneficial for burn victims as there would be no additional disinfecting of the wound required once the artificial skin is removed.

The curvatures of the human body are also another consideration when designing the exact area for the 3D printed skin. It is important to consider the tolerances and the limitations of the 3D printer when the measurements are taken. Each printed skin has to be designed specifically for that user. The edges of the skin have designed to be rounded and not sharp so that it is safer for the user to use.

\section{References}

1. M. W. Michell, H. M. Oliveira, M. P. Kinsky, S. U. Vaid, D. N. Herndon, and G. C. Kramer, J. Burn Care Res. 27, 6, pp. 819-825 (2006)

2. M. Bezuhly and J. S. Fish, Plast. Reconstr. Surg. 130, 2, pp. 349e-358e (2012)

3. D. Church, S. Elsayed, O. Reid, B. Winston, and R. Lindsay, Clinical Microbiology Reviews 19, 2, pp. 403-434 (2006)

4. Konya C, Sanada H, Sugama J, et al. J Clin Nurs 9, pp. 1236-42 (2010)

5. T. Lefrancois, K. Mehta, V. Sullivan, S. Lin, M. Glazebrook, Foot Ankle Surg 23, 4, pp. 215-224 (2016)

6. A. N. I. D. Gantry, K. Johnson, A. Dubey, C. Harris, and D. Sasaki, Radiother. Oncol 120, pp. S33-S34 (2016)

7. R. Ricotti et al., Phys. Medica 32, 12, pp. 1698-1706 (2016)

8. W.-K. Lee, Y.-Y. Lim, A. T.-C. Leow, P. Namasivayam, J. Ong Abdullah, and C.-L. Ho, Carbohydr. Polym 164, pp. 23-30 (2017)

9. S. Hsieh, H. H. Chou, C. W. Hsieh, D. C. Wub, C. H. Kuo, and F. H. Lin, Food Chem 141, 3, pp. 2117-2121 (2013) 